\title{
Pituitary Apoplexy Causing Compression of Third Cranial Nerve-Management
}

\author{
Václav Masopust ${ }^{1}$ \\ ${ }^{1}$ Department of Neurosurgery, First Faculty of Medicine, Central \\ Military Hospital, Charles University, Prague, Czech Republic
}

\begin{abstract}
Address for correspondence Václav Masopust, MD, PhD, LLM, MBA, Department of Neurosurgery, Central Military Hospital, U vojenské nemocnice 1200, Prague 16200, Czech Republic (e-mail: masopust@uvn.cz).
\end{abstract}

\begin{abstract}
Keywords

- pituitary apoplexy

- oculomotor nerve

- anatomical variations

- sella turcica
\end{abstract}

\section{Introduction}

Pituitary apoplexy is caused by an acute ischemic stroke, with secondary bleeding after the stroke, or it could be due to a primary bleeding event in pituitary adenomas. Some evidence indicates that pituitary apoplexy is associated with the sudden onset of neurological and/or hormonal disorder. ${ }^{1}$ One possible neurologic symptom is paresis/plegia of the third cranial nerve. Lesions of the third cranial nerve are rare. However, the possible cause of a lesion of the oculomotor nerves passing through the cavernous sinus as well as the trochlear and abducens nerves and visual acuity deficits may be an acute onset of pressure on the cavernous sinus. However, the most common nerve lesion is the oculomor nerve with varying degrees of paresis. ${ }^{2}$ The nerve lesion of the oculomotor nerve is very rare (14.28\% from all pituitary apoplexy), though the incidence in the patient group is relatively high and the formation of this nerve lesion has always been associated with pituitary tumor apoplexy (100\%). ${ }^{3}$ Possible causes of the nerve lesion and anatomical variations are discussed by the authors.

\section{Materials and Methods}

From April 2011 to August 2012, 129 patients underwent surgery for pituitary adenoma at the Department of Neurosurgery, First Medical Faculty and Central Military Hospital, Military Faculty Hospital, Prague. The group of patients comprised 62 women and 67 men, with a mean age of 52.5 years (range: 9-81 years). In the patient group, there were 51 hormonally active tumors and 78 were hormonally inactive (requiring surgery for visual disorder or for compression of the surrounding structure). Four patients (3.1\%) presented lesions of the oculomotorius nerve-complete oculomotor received

June 9, 2018

accepted

August 6, 2018

published online

August 27, 2019
Dol https://doi.org/

$10.1055 / \mathrm{s}-0039-1694849$

ISSN 2277-954X.
(C2019 Neurological Surgeons'

Society of India
License terms

()ㅜ(1) $\Theta \circledast$ 
nerve palsy (ptosis, diplopia, and mydriasis). In this group of four patients (mean age: 55 years, range: 36-65 years), all patients (two women and two men) underwent surgery. Early clinical symptoms appeared anywhere from 1 to 3 weeks before the surgery. The appearance of lesions of the oculomotor nerve was sudden in all cases. One patient suffered from panhypopituitarism preoperatively. The tumor size varied from 18 to $23 \mathrm{~mm}$ (diameter $20 \mathrm{~mm}$ ). In one case, a tumor penetration into the cavernous sinus was demonstrated based on preoperative magnetic resonance imaging (MRI). Disintegrating portion of tumor and marks of bleeding were typically presented on the MRIs. In two patients, the arrested pneumatization and thickened bone were observed on MRI. In the remaining two patients, a thickened diaphragm was more easily noted after the_removal of the tumor in the control MRI scan the next day.

Since November 2006, our team has used the binostril endoscopic endonasal approach with the aid of a Storz 0 degree endoscope (Karl Storz Endoscope). The binostril technique (four-hand technique) is the standard surgical technique in our department. This procedure does not require septum lateralization. We resect only a small area $(0.5 \times 0.5 \mathrm{~cm})$ in the posterior part of the nasal septum. The use of both nostrils offers several advantages, including greater working space and a large range of instrument movement. At a certain point during the surgical procedure, two instruments have to be used simultaneously, namely, a drain cannula and an endoscope (hence, the four-hand technique). Our operations take place in a multifunction room, where data are transmitted from an intraoperative 3.0-TMR system (General Electric) and subsequently updated in a surgical navigation system (Brainlab).

\section{Results}

In two cases, there was the absence of the sphenoid cavity, as well as evidence of thickened bone. In these two cases, it was necessary to grind the bone (width from $6 \mathrm{~mm}$ to $1 \mathrm{~cm}$ ) (-Fig. 1). A very solid opaque diaphragm was macroscopically

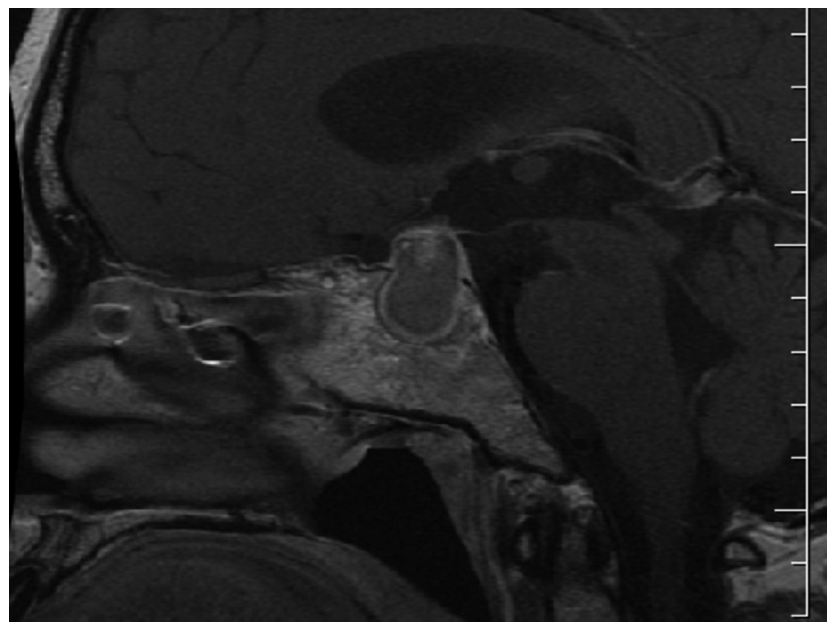

Fig. 1 Arrested pneumatization, as well as evidence of thickened bone on magnetic resonance image. visible after the removal of the tumor and thickened bone in the remaining two cases (-Fig. 2). The diaphragm was whitish, smooth, and strong in appearance. There was no typical decline of the diaphragm in these two cases after the removal of the tumor. A tougher, but fragile, bruising tumor with initial high pressure was found in all cases after cutting the dura mater. After cutting the dura mater, there was always a massive sharp leakage of old blood as a part of the sellar expansion. All patients showed a very fast recovery. Partial recovery generally occurred during the first 24 hours. Complete adjustment was observed in all patients within 1 week after the surgery. MR radical resection (3 months postoperatively) and hormonal radical resection (for hormonal active tumors) were achieved in $73 \%$ of the cases with the help of intraoperative MRI (129 operations for pituitary adenomas in all patients). Magnetic resonance (MR) radical resection of a subgroup of patients (the subgroup consisted of adenomas with lesions of the oculomotor nerve) was achieved in three adenomas, $75 \%$. On postoperative MRI, we observed the remains of the left side of the adenomas in the lateral compartment of the cavernous sinus. We did not encounter any postoperative complications in the cases with lesions of the oculomotor nerve. Pituitary hypofunction remained in patients with substituted hypofunctional hypophysis before surgery. Postoperative substitution has been necessary in two patients, 5 years after the surgery. Histologically, bruising was seen in all of the adenomas.

\section{Discussion}

Pituitary apoplexy is caused by acute ischemic injury or spontaneous hemorrhage of the pituitary tumors. The frequency of stroke ranges from 2 to $7 \%{ }^{4}$ However, it is important to determine whether it is a clean bleeding, which is shown on MR, or clinically developed clinical symptoms (sudden malfunction of the pituitary gland, visual disturbances, or disorders of the ocular nerves). The increased fragility of tumor vessels has been suggested as a cause of pituitary apoplexy. ${ }^{1}$ The embolism in atherosclerotic disease

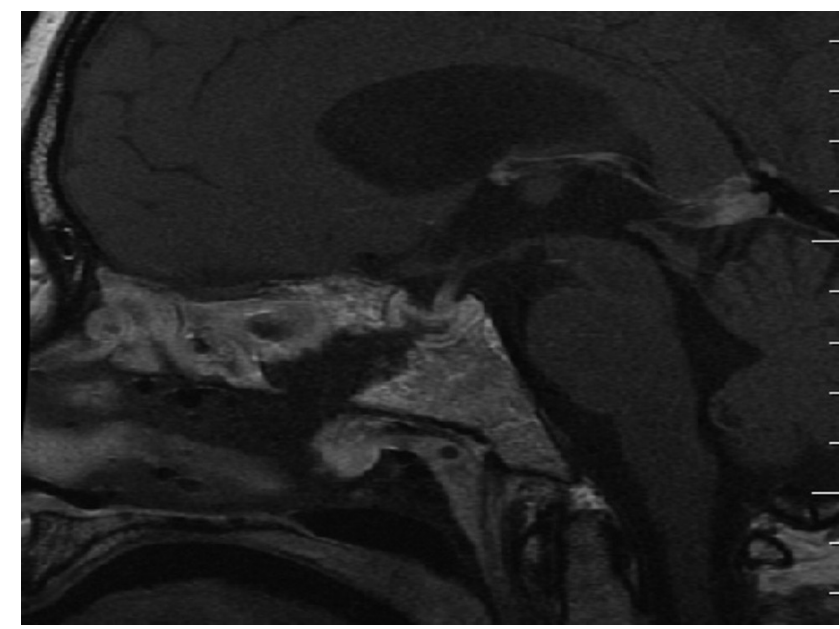

Fig. 2 Very solid opaque diaphragm on magnetic resonance image. 
has been also suggested as a cause of pituitary apoplexy. ${ }^{4}$ Predisposing factors of pituitary apoplexy include high blood pressure, head injury, pituitary irradiation in the past, anticoagulant therapy, treatment with dopamine agonists and pregnancy, diabetes mellitus, high altitude, dengue hemorrhagic fever, dynamic endocrine tests, and spinal anesthesia. ${ }^{5,6}$ The latter factor can also support the hypothesis that the rapid enlargement of pituitary adenoma may lead to the oppression of veins, which are a part of the portal system in the pituitary stalk through diaphragm. This condition can lead to congestion and hemorrhage ${ }^{7}$; however, small tumors can also develop apoplexy events. ${ }^{3}$ Intratumoral vasculopathy rendering the vessels more susceptible to hemorrhage has been proposed as an option. ${ }^{3}$ Furthermore, a significant correlation between the intratumoral expression of vascular endothelial growth factor and tumor necrosis factor- $\alpha$ and the presence of tumor hemorrhage has been found, suggesting a possible causal relationship, although other group did not confirm this finding. ${ }^{3}$

Lesions of the oculomotor nerve that result from pituitary apoplexy can have several causes. The notion of direct pressure on a nerve by a large pituitary adenoma is apparently the most logical cause. ${ }^{89}$ Pituitary apoplexy is an acute condition in which the wall of the cavernous sinus is not primarily infiltrated yet is enhanced pressure inside the sinus. The conjecture pertaining to vascular lesions of the oculomotor nerve, which is based on a reflex spasm of vessels from the pressure of the tumor that encases the internal carotid artery, is much more intriguing. ${ }^{10}$ This belief explains the emergence of acute lesions, but cannot explain why it would be limited only to the disability of the oculomotor nerve and not to the trochlear and abducens nerves. ${ }^{11}$ Presently, the theory that is best is still being debated. The basic premise is that the oculomotor nerve, which passes through the lateral wall of the cavernous sinus, is much more vulnerable than the other nerves. It has been suggested that the abducens nerve is more vulnerable than the oculomotor nerve. The cause of the sixth cranial nerve lesion is beyond the cavernous sinus and this lesion is described more often in relation to either an inflammation or to a skull base tumor. ${ }^{12}$ The highest position of the third cranial nerve (the oculomotor nerve) in cavernous sinus is another explanation as to why the nerve is affected. The anterior part of the cavernous sinus is relatively the most mobile part. ${ }^{13}$

We believe that the most likely cause of the disability of the oculomotor nerve is the anatomy of the sella turcica and its surroundings. The frontal, caudal, and dorsal surfaces are made up of bone. The cranial saddle surfaces are covered with the diaphragm. The lateral surface forms the inner walls of the cavernous sinus. The pressure is transmitted to the wall of the cavernous sinus if the diaphragm is very rigid, or if the base of the saddle is rigid. The cavernous sinus is not an open tube resembling an enlarged vein, but rather it is a patchwork of lagoons (sometimes described as spicules, which form a dense network). This structure resembles a sponge, with high blood flow. ${ }^{14}$ Congestion in the sella turcica leads to high pressure inside the cavernous sinus. The oculomotor nerve is located uppermost, above the trochlear nerve in the lateral wall of the cavernous sinus at the transition to the upper surface. These conditions explain why the highest pressure is at this particular point. The conditions also explain why the site of the pressure is at the lesion of the oculomotor nerve.

This hypothesis is confirmed by our results. All operations were performed by the four-hand endoscopic technique through two nostrils (standard procedure at our institution). ${ }^{15}$ In all patients, perioperative MRI was applied at the same time. The maximum radicality of tumor removal is guaranteed by this technique at this time. ${ }^{16}$ No complications were seen in any of our patients. All patients were transmitted by car to the Department of Endocrinology at the Charles University Hospital in Prague.

As our subgroup consisted of only four patients, no statistical analyses could be done. Nevertheless, we found some evidence to support our hypothesis. The most important finding concerns the underdevelopment of the sphenoid sinus with a solid saddle base that was seen in two patients (conchal type of sphenoid sinus). In the remaining two patients, we observed a very strong diaphragm that became visible through MRI after tumor removal and thickened bone (presellar type of sphenoid sinus). We found anatomical variety in all patients with lesions of the third carinal nerve. This observation is a new concept which was found. These findings suggest that the primary pressure is at the lateral wall of the cavernous sinus. The incidence of presellar type was reported to be 5.5 to $27 \%$ in the literature and conchal type is reported to be none to $3 \% .^{17-19}$

In the whole group of 129 patients (including hormonally active microadenomas), the average tumor size was $21 \mathrm{~mm}$; in the subgroup (lesions of the oculomotorius nerve), tumor size ranged from 18 to $23 \mathrm{~mm}$ (diameter $20 \mathrm{~mm}$ ). These findings suggest that lesion formation is not determined by the size of the tumor, but rather by the speed of the pressure buildup in the event that anatomical variations are present where the pressure is directed toward the cavernous sinus.

The hypothesis that pressure is the cause of the oculomotor nerve lesion was confirmed by the rapid speed of adjustment, that is, all patients demonstrated partial modification in the first 24 hours after the surgery. Moreover, all patients were able to lift their eyelids on the first postoperative day.

\section{Conclusion}

Early tumor resection is the treatment of choice for lesions of the oculomotor nerve, which are caused by pituitary apoplexy. The hypothesis that sudden pressure causes oculomotor nerve lesions is highly plausible in the event that anatomical variations are present, but this could not be demonstrated statistically in the present report because of the small number of cases.

\section{Funding}

This study was funded by grant IGA MZ NT 13631 and by grant PROGRES Q35. 


\section{Conflicts of Interest}

Václav Masopust has received research grants IGA MZ NT 13631 from the internal grant agency of Czech Republic and PROGRES Q35 from the Charles University. David Netuka has received research grant IGA MZ NT 13631 from the internal grant agency of Czech Republic.

\section{References}

1 Cardoso ER, Peterson EW. Pituitary apoplexy: a review. Neurosurgery 1984;14(3):363-373

2 Murad-Kejbou S, Eggenberger E. Pituitary apoplexy: evaluation, management, and prognosis. Curr Opin Ophthalmol 2009;20:456-461

3 Solomon AE, Tataranu L, Ciubotaru V, Gorgan MR. Pituitary apoplexy: clinical features, management and outcome. Clinical study and review of the literature. Romania Neurosurgery 2015;29:68-76

4 Singh TD, Valizadeh N, Meyer FB, Atkinson JLD, Erickson D, Rabinstein AA. Management and outcomes of pituitary apoplexy. J Neurosurg 2015;122(6):1450-1457

5 Murad-Kejbou S, Eggenberger E. Pituitary apoplexy: evaluation, management, and prognosis. Curr Opin Ophthalmol 2009;20(6):456-461

6 Capatina C, Inder W, Karavitaki N, Wass JA. Management of endocrine disease: pituitary tumour apoplexy. Eur J Endocrinol 2015;172(5):R179-R190

7 Semple PL, Jane JA Jr. Laws ER Jr. Clinical relevance of precipitating factors in pituitary apoplexy. Neurosurgery 2007;61(5):956-961, discussion 961-962

8 Sussman EB, Porro RS. Pituitary apoplexy: the role of atheromatous emboli. Stroke 1974;5(3):318-323
9 Lenthall RK, Jaspan T. A case of isolated third nerve palsy with pupil involvement. Br J Radiol 2000;73(869):569-570

10 Saul RF, Hilliker JK. Third nerve palsy: the presenting sign of a pituitary adenoma in five patients and the only neurological sign in four patients. J Clin Neuroophthalmol 1985;5(3):185-193

11 Rossitch E Jr. Carrazana EJ, Black PM. Isolated oculomotor nerve palsy following apoplexy of a pituitary adenoma. J Neurosurg Sci 1992;36(2):103-105

12 Merino P, Gómez de Liaño P, Villalobo JM, Franco G, Gómez de Liaño R. Etiology and treatment of pediatric sixth nerve palsy. J AAPOS 2010;14(6):502-505

13 Martins C, Costa E Silva IE, Campero A, et al. Microsurgical anatomy of the orbit: the rule of seven. Anat Res Int 2011;2011:468727

14 Hemza J, Biomechanical Problems of Thin-Walled Veins of the Brain. Prague: Department of Anatomy and Biomechanics of Charles University, Faculty of Physical Education and Sport 2010

15 Masopust V, Neutka D, Beneš V. Endoscopic endonasal transsphenoidal resection of sellar lesions. Czech and Slovak Neurology and Neurosurgery 2008;104:704-710

16 Netuka D, Masopust V, Beneš V. Treatment of pituitary adenomas. Czech and Slovak Neurology and Neurosurgery 2011;107:240-253

17 Baskin JZ, Kuriakose MA, Lebowitz RA. The anatomy and physiology of the sphenoid sinus. Oper Tech Otolaryngol 2003;14:168-172

18 Hamid O, El Fiky L, Hassan O, Kotb A, El Fiky S. Anatomic variations of the sphenoid sinus and their impact on trans-sphenoid pituitary surgery. Skull Base 2008;18(1):9-15

19 Sevinc O, Is M, Barut C, Erdogan A. Anatomic variations of sphenoid sinus pneumatization in a sample of Turkish population: MRI study. Int J Morphol 2014;32:1140-1143 